\title{
Public interest in predictive genetic testing, including direct-to-consumer testing, for susceptibility to major depression: preliminary findings
}

\begin{abstract}
Alex Wilde ${ }^{\star 1,3}$, Bettina Meiser ${ }^{2}$, Philip B Mitchell ${ }^{1,3,6}$ and Peter R Schofield ${ }^{4,5,6}$
The past decade has seen rapid advances in the identification of associations between candidate genes and a range of common multifactorial disorders. This paper evaluates public attitudes towards the complexity of genetic risk prediction in psychiatry involving susceptibility genes, uncertain penetrance and gene-environment interactions on which successful molecular-based mental health interventions will depend. A qualitative approach was taken to enable the exploration of the views of the public. Four structured focus groups were conducted with a total of $\mathbf{3 6}$ participants. The majority of participants indicated interest in having a genetic test for susceptibility to major depression, if it was available. Having a family history of mental illness was cited as a major reason. After discussion of perceived positive and negative implications of predictive genetic testing, nine of 24 participants initially interested in having such a test changed their mind. Fear of genetic discrimination and privacy issues predominantly influenced change of attitude. All participants still interested in having a predictive genetic test for risk for depression reported they would only do so through trusted medical professionals. Participants were unanimously against direct-to-consumer genetic testing marketed through the Internet, although some would consider it if there was suitable protection against discrimination. The study highlights the importance of general practitioner and public education about psychiatric genetics, and the availability of appropriate treatment and support services prior to implementation of future predictive genetic testing services.
\end{abstract}

European Journal of Human Genetics (2010) 18, 47-51; doi:10.1038/ejhg.2009.138; published online 19 August 2009

Keywords: predictive genetic testing; psychiatric genetics; major depression; direct-to-consumer genetic testing; public opinion; mental health

\section{INTRODUCTION}

The identification of candidate genes thought to confer susceptibility to psychiatric illness, manifesting on exposure to stressful life events, presents an opportunity to predict high-risk groups and reduce the burden of psychiatric disease through intervention strategies at a presymptomatic stage. Risk prediction and preventative interventions, based on first episode psychosis, are already in place for youth at high risk for schizophrenia and other psychotic disorders. Studies show that early interventions in this group, such as pharmacotherapy and psychotherapy, may delay or even prevent progression to a diagnosable psychotic disorder such as schizophrenia. Predictive genetic testing for markers of mental illness has thus far not been studied in prospective early intervention studies. ${ }^{1}$ Effective interventions that use genetic and environmental risk information will depend on public understanding of the complexity of interactions between susceptibility genes of uncertain penetrance and environmental risk factors. The recent proliferation of commercial start-up genetic testing companies marketing predictive genetic tests directly to the public has raised concerns about predictive validity and potential health impact of such tests. $^{2}$ Consumers may be at risk of selecting inappropriate tests, misinterpreting results and making harmful health decisions. ${ }^{3}$ Private biotechnology laboratories, based predominantly in USA, UK and Iceland, are currently marketing more than 50 predictive genetic tests direct-to-consumer (DTC) through the Internet. Some of these tests are available for use in clinical practice, but for some, including the susceptibility tests for psychiatric disorders, there are few published data thus far to support clinical validity. ${ }^{4}$

A gene-disease susceptibility association that has been replicated by a large number of studies is an interaction between a functional polymorphism in the promoter region $(5-H T T L P R)$ of the serotonin transporter gene (SCL6A4) and exposure to stressful life events in increasing the likelihood of a major depression in non-clinical populations of adults, ${ }^{5-8}$ adolescents ${ }^{9}$ and children. ${ }^{10}$ The evidence suggests that individuals homozygous for the short allele $(\mathrm{s} / \mathrm{s})$ of the serotonin transporter gene-linked polymorphic region may be at an increased risk for depression on exposure to multiple stressful life events. ${ }^{5-10}$ Since the completion of this study, a recent meta-analysis has found no evidence for a $5-H T T L P R$ by environment interaction in association with an increased risk of depression, ${ }^{11}$ however, 5-HTTLPR genotyping remains a good hypothetical example by which to evaluate attitudes to predictive genetic testing for a risk for a common complex disorder.

${ }^{1}$ School of Psychiatry, University of New South Wales, Sydney, NSW, Australia; ${ }^{2}$ Prince of Wales Clinical School, University of New South Wales, Sydney, NSW, Australia; ${ }^{3}$ Black Dog Institute, Sydney, NSW, Australia; ${ }^{4}$ Prince of Wales Medical Research Institute, Sydney, NSW, Australia; ${ }^{5}$ School of Medical Sciences, University of New South Wales, Sydney, NSW, Australia; ${ }^{6}$ Brain Sciences UNSW, Sydney, NSW, Australia

*Correspondence: A Wilde, School of Psychiatry, University of New South Wales, Black Dog Institute Building, Prince of Wales Hospital, Hospital Road, Randwick, NSW 2031, Australia. Tel: +61 29382 8511; Fax: +61 29382 8151; E-mail: alex.wilde@unsw.edu.au

Received 8 January 2009; revised 10 July 2009; accepted 15 July 2009; published online 19 August 2009 
Psychosocial issues associated with predictive genetic testing for susceptibility to major depression are likely to be more complex than for Mendelian monogenic disorders because test results are not definitive. There will be implications for public policy and ethics with an impact on stigma ${ }^{12-15}$ and concerns about potential misuse of genetic risk information, for example, through employment and insurance discrimination. ${ }^{16}$

Government genetics advisory bodies around the world have commenced expert consultations and public meetings to determine how predictive genetic testing should be regulated, ${ }^{3,17,18}$ however, there is a dearth of scientific research to inform national and international policy.

Previous international scientific research in this area is predominantly limited to preliminary and/or qualitative studies on attitudes towards genetic risk information and genetic testing among members of families who have multiple relatives affected by bipolar disorder or schizophrenia. ${ }^{12,19-22}$ These studies have generally found positive attitudes towards predictive genetic testing, and a recent quantitative study involving families with a high density of bipolar disorder showed that interest in testing increased with the certainty indicated by the test. ${ }^{23}$ As no research to date has evaluated attitudes among the general population towards predictive genetic testing for depression risk and beliefs about the psychosocial implications, this study aimed to qualitatively assess public understanding of, and attitudes towards, risk prediction involving susceptibility genes for depression based on 5-HTTLPR genotyping.

\section{MATERIALS AND METHODS}

The results reported here were undertaken as part of a broader qualitative study, which also explored attitudes towards preventative mental health interventions based on genetic risk, ${ }^{24}$ as well as the perceived impact of media portrayal of genetics and mental illness. These findings will be reported separately.

As this is a relatively unexplored area of enquiry, a qualitative methodology was used. There has been an upsurge in interest in studies that examine attitudes, beliefs and experiences of people in connection to health care issues, and qualitative methodology has been increasingly recognised by evidencebased clinical researchers. ${ }^{25}$

\section{Participants}

A market research company was engaged to randomly recruit 10 participants each to four or more focus groups from their database of 10000 members of the public unselected for disease risk, ensuring an even mix of gender, age and socio-demographic backgrounds. Eligibility criteria included being 18 years or older, fluent in English, residing in the Sydney metropolitan area and should not have participated in any research in the previous six months. Ethical approval for the study was provided by the relevant Institutional Review Board (Human Research Ethics Committee, University of New South Wales, Australia).

\section{Focus group interviews}

Participants were naive to the focus group topic. They completed a short questionnaire that included items about age, sex, language spoken at home, occupation and highest education level completed. Participants were asked to introduce themselves and indicate whether they had prior knowledge or experience of the subject of mental illness. They were not required to disclose personal or family history of mental illness.

The focus groups were formed in accordance with widely accepted standards of focus group methodology. ${ }^{26}$ They were facilitated by the first author (a research scientist and medical journalist) and observed by the second author (a research psychologist). Focus groups were recorded on digital video and the observer took written notes of the main themes discussed.
An interview guide was developed on the basis of a review of the relevant literature with input from all authors exploring the following topics: (i) interest in predictive genetic testing (genotyping for the $5-H T T$ promoter polymorphism) to determine susceptibility to major depression and (ii) attitudes towards potential for social stigma, discrimination and issues of DNA privacy.

Genotyping for the 5-HTT promoter polymorphism was framed to participants as a 'genetic test to determine whether an individual has an increased risk for developing depression in the event of experiencing significant adversity'. A test result showing the s/s (higher risk) genotype of the 5-HTT promoter polymorphism was framed to participants as a genetic result indicating an 'increased risk for depression'.

\section{Analysis}

The conceptual approaches of Patton, ${ }^{27}$ and Miles and Huberman ${ }^{28}$ were used as guides for the analysis. A detailed coding scheme was developed and transcripts were coded by the first author. Ten percent of the transcripts were recoded by the second author, to identify any discrepancies in the interpretation of codes and refine the coding scheme. Discrepancies were discussed between coders to provide opportunities for developing further coding and consensus. ${ }^{25}$

Coded transcripts were subsequently analysed for emergent themes with the assistance of the software package QSR N6 (Qualitative Solutions and Research, Doncaster, Victoria, Australia), which facilitated comparisons between affected and unaffected participants as well as other aspects of the analysis. ${ }^{28}$

Corresponding to the qualitative nature of the data, focus group discussions were designed to identify the range of beliefs rather than extent to which participants held particular beliefs. However, to provide an indication of the extent of interest in predictive genetic testing for susceptibility to depression, every participant was asked whether they would undergo genotyping for the 5-HTT promoter polymorphism if it was available, and why, before and after discussion of perceived positive and negative psychosocial implications.

As the aim of the study was to evaluate attitudes towards predictive genetic testing among individuals for whom such testing would carry greater hereditary implications compared with those without a personal or family history, participant quotations were coded according to lived experience (personal and familial implications) of mental illness: eg [A] (affected): reported personal or family history of major depression, bipolar disorder or schizophrenia; or [U] (unaffected): no reported personal or family history of major depression, bipolar disorder or schizophrenia. Interest in genetic testing was also coded: [YY], interested in having a genetic test for susceptibility to major depression both before and after considering implications; $[\mathrm{YN}]$, initially interested in having the genetic test but not after considering implications; $[\mathrm{NN}]$, not interested in having the genetic test both before and after considering implications. Although an NY code was a theoretical possibility, it was not used because no participants fell into this category.

\section{RESULTS}

\section{Participation and demographics}

Thirty-six people (18 female, 18 male) participated in four focus groups (8-10 people per group) held at four locations across Sydney. Recruitment was discontinued after informational redundancy was achieved in the fourth focus group, in accordance with standard qualitative methodology. ${ }^{28}$ During focus group discussions, 14 participants spontaneously revealed a personal or family history of major depression, bipolar disorder or schizophrenia. Demographic characteristics of affected and unaffected participants are shown in Table 1. The mean age was 41 (range 20-65 years).

\section{Interest in predictive genetic testing for 5-HTTLPR genotype}

At the beginning of the discussion, the majority of participants (10 affected, 14 unaffected) indicated an interest in undergoing a genetic test for susceptibility to major depression, if the test was available. Unaffected participants who said they would be interested in having predictive testing were more hesitant and tended to attach conditions. Table 2 shows interest in undergoing 5-HTTLPR genotyping before and after discussion of perceived positive and negative implications, 
Table 1 Demographic characteristics of the sample

\begin{tabular}{|c|c|c|c|c|}
\hline \multirow[t]{2}{*}{ Variable } & \multirow{2}{*}{$\begin{array}{l}\text { Affected }(A)^{\mathrm{a}} \\
\qquad(\mathrm{N}=14) \\
\mathrm{N}\end{array}$} & \multicolumn{2}{|c|}{ Unaffected $(U)^{\mathrm{b}}$} & \multirow{2}{*}{$\begin{array}{l}\text { Total samplec } \\
\qquad(\mathrm{N}=36) \\
\mathrm{N}\end{array}$} \\
\hline & & & & \\
\hline \multicolumn{5}{|l|}{ Sex } \\
\hline Male & 5 & \multicolumn{2}{|c|}{13} & 18 \\
\hline Female & 9 & \multicolumn{2}{|c|}{9} & 18 \\
\hline \multicolumn{5}{|l|}{ Age (years) ${ }^{c}$} \\
\hline $18-29$ & 2 & \multicolumn{2}{|c|}{5} & 7 \\
\hline 30-39 & 6 & \multicolumn{2}{|c|}{6} & 12 \\
\hline $40-49$ & 1 & \multicolumn{2}{|c|}{3} & 4 \\
\hline $50-59$ & 4 & \multicolumn{2}{|c|}{2} & 6 \\
\hline $60-69$ & 1 & \multicolumn{2}{|c|}{5} & 6 \\
\hline \multicolumn{5}{|c|}{ Highest education level completed ${ }^{\mathrm{c}}$} \\
\hline Tertiary & 9 & \multicolumn{2}{|c|}{9} & 18 \\
\hline High school & 5 & \multicolumn{2}{|c|}{12} & 17 \\
\hline \multicolumn{5}{|c|}{$\begin{array}{l}\text { aSelf-reported personal or family history of major depression, bipolar disorder or schizophrenia. } \\
\text { bNo reported personal or family history of major depression, bipolar disorder or schizophrenia. } \\
\text { cMissing value - participant declined questions related to age and education. }\end{array}$} \\
\hline \multirow[t]{2}{*}{ Interest } & & \multicolumn{3}{|c|}{ Participants } \\
\hline & & Affected & $\begin{array}{l}\text { Unaffected } \\
\text { (unsure) }^{a}\end{array}$ & $\begin{array}{c}\text { Total } \\
\text { (unsure) }^{a}\end{array}$ \\
\hline Initially interes & & 10 & $14(2)$ & $24(2)$ \\
\hline No longer inte & after discussion & 4 & 5 & 9 \\
\hline Still interested & discussion & 6 & $7(4)$ & $13(4)$ \\
\hline
\end{tabular}

aparticipants who were unsure of whether they would undergo such a test.

and reading several media articles about various aspects of genes and mental illness. Participants who were initially interested in having 5-HTTLPR genotyping but who changed their mind after the discussion, citied fear of genetic discrimination and loss of privacy as major reasons. No participants changed their mind in the opposite direction.

Perceived benefits of predictive testing for 5-HTTLPR genotype Benefits for families. Both affected and unaffected participants thought predictive testing for susceptibility to depression would be of greater benefit to those with a family history of the disorder.

'I couldn't imagine having the test unless there was somebody in the family with mental illness' [A/YY].

Scope for early intervention. Both affected and unaffected participants thought 5-HTTLPR genotyping would help them be ready to seek early help. One remarked: '...forewarned is forearmed', which he believed would enable him to ' ... deal with it should it arise' [U/YY].

Another said:

'...I've seen my mum live through it, I think it's so much better to know straight out, start as soon as you can with whatever help you can get.' [A/YY].

One participant suggested 5-HTTLPR genotyping could be a useful part of a general health check-up [A/YY]. One participant said knowledge of one's genetic risk could help people put techniques in place that might minimise or prevent the development or severity of depression. [A/YN].
Reduce social stigma. Several affected participants thought evidence of a genetic component would help validate depression and other mental illnesses as physical illnesses, which might decrease social stigma. One suggested this would lead to improved government funding for mental health research. Another disagreed with predictive genetic testing for susceptibility to depression because 'the test is not definitive', and 'no prevention is available.'

Conditions attached to interest in predictive genetic testing. Conditions set by unaffected participants interested in having 5-HTTLPR genotyping included: 'if it ran in the family;' [U/NN], 'if I needed it', [U/YY], 'if the doctor referred me,' [U/YY]. One participant saw little point in having a predictive genetic test without availability of related interventions:

'You'd just wait for the signs of symptoms to come. Nothing is going to change; you don't start taking something just because there's a possibility you might [develop depression].' [U/NN].

\section{Perceived disadvantages of predictive genetic testing for $5-H T T L P R$ genotype}

Fear of loss of privacy. Although most participants said they trusted a genetic test result would remain private and confidential if obtained through the public health system, some participants were worried that privacy could not be guaranteed. One participant cited this as the reason why she would not undergo 5-HTTLPR genotyping:

' ... if [the test result] fell into the wrong hands or ... you know we just live in such a fish bowl these days and no, couldn't bear the thought of it.' [U/NN].

Risk of discrimination. Many participants were concerned that undergoing 5-HTTLPR genotyping could lead to discrimination by insurance companies and employers; which influenced several unaffected participants against undergoing a predictive test, and caused another to change her mind:

'I know that if I had a test well it probably would come back positive. And if found that out and I couldn't get insurance well then I'd say no to a test.' [A/YN].

Risk of fatalistic thinking. Both affected and unaffected participants thought they might develop fatalistic thinking if they were found to have the 5-HTTLPR s/s variant:

'...once you find out...that you are in this predisposition it might send you over the mark ... you'd be worrying the whole time - that's going to cause it.' [A/YN].

One participant viewed having the $s / s$ variant as definitive with negative consequences:

'I mean you might be okay and then it comes back that you're depressed or you've got bipolar [disorder] and then you go and neck yourself.' [U/YN].

One participant disagreed with the fatalistic view, and emphasised the importance of awareness:

'I'd be worried if I wasn't aware... if it's $80 \%$ risk or something like that at least I know, I'm aware that this could happen. I'm not going to treat it as if it is happening.' [A/YY].

Increase social stigma. Several affected and unaffected participants anticipated that predictive testing for predisposition to depression would not reduce social stigma attached to the disorder but could increase it: 'Whilst I see that [predictive genetic testing] might be valuable to helping a person... I think social implications, social stigma is the major problem.' [A/YY]. 
Attitudes towards DTC predictive genetic testing marketed through the Internet

Participants were told that DTC predictive genetic testing thought to determine predisposition to depression in consort with environmental risk factors involved registering online and sending a saliva sample or cheek swab to an overseas genetic testing laboratory in a DNA test kit provided. All 26 participants who responded to this issue were unanimously against accessing DTC predictive genetic testing from biotechnology companies. Objections included concern about credibility of DTC genetic testing services, especially if obtained through the Internet, worries with regard to the security of DNA sample and privacy of genetic risk information, and lack of confidence in non face-to-face genetic counselling.

\section{DISCUSSION}

\section{Interest in predictive genetic testing for 5-HTTLPR genotype}

This study found positive attitudes towards predictive genetic testing associated with susceptibility to major depression if it were to become available, which supports previous findings for bipolar disorder or schizophrenia. ${ }^{12,15,22,23,29}$ The results suggest having a personal or family experience of depression, bipolar disorder or schizophrenia may be a strong predictor of uptake of predictive genetic testing for mental disorders. As the National estimated lifetime risk for mental illness is estimated to be $20-25 \%$, it is expected that a proportion of a population sample would report personal or family experience of depression or other mental disorders.

Perceived discrimination by insurers or employers and perceived risks to security of genetic information seemed to moderate interest in predictive genetic testing among both affected (having a personal or family history of a mental disorder) and unaffected individuals. Similar concerns were described in a study of attitudes towards predictive genetic testing for susceptibility to schizophrenia. ${ }^{22}$

The majority of participants who were interested in having the hypothetical test said they would still have it despite the result offering a probabilistic rather than a definitive risk. These findings support a previous study on families with a high density of bipolar disorder, which revealed a comparably higher degree of perceived disadvantages of a probabilistic risk versus certainty of risk. ${ }^{12}$ It could be that members of families with a high frequency of bipolar disorder perceive uncertain risk to exert a greater burden than do affected or unaffected members of the public.

The majority of unaffected participants who were interested in having a hypothetical predictive test for susceptibility to depression tended to cite conditions under which they would have the test, whereas affected participants did not. This suggests having a personal or family experience of a mental illness may engender a greater amenability towards 5-HTTLPR genotyping. These attitudes may be influenced by naivety about low predictive power of such tests and low risk rates for close family members. Potential differences in attitude and approach to hypothetical predictive genetic testing between affected and unaffected individuals should be considered when planning molecular-based mental health interventions and public education about predictive testing for susceptibility to a psychiatric disorder. Further studies are required to find out whether these trends are borne out in larger non-clinical samples.

\section{Interest in direct-to-consumer predictive genetic testing}

To our knowledge no previous studies have evaluated public interest in the emerging area of DTC predictive genetic testing. Although unanimous opposition to DTC predictive genetic testing for depression risk alleles in the present study suggested low potential uptake of commercial genetic testing, minor interest was restored if protection against discrimination and DNA misuse could be guaranteed. Austin et $a l^{29}$ anticipated that the availability of DTC testing for psychotic disorders would justify making psychiatric genetic counseling routinely available. Participants' trust in the public health system as a potential provider of predictive genetic testing and counseling seen in this study suggests, as publicity for DTC genetic testing increases, there could be an unreasonable demand on general practitioners to interpret the results of genetic tests they have not ordered and are not trained to interpret. A large quantitative population study will be necessary to assess attitudes towards DTC genetic testing in a representative population and potential demand for genetic counseling.

\section{Perceived impact of predictive genetic testing on stigma}

Theories exist that a biological component for a mental illness shifts responsibility away from self to one's biology, thus reducing the blame and consequently the stigma associated with these disorders. ${ }^{13,30,31}$ Conversely empirical evidence suggests a genetic model for mental illness may increase the perceived seriousness of these disorders and increase stigma. ${ }^{15,30,31}$ These findings are further supported by a study that found endorsement of genetic explanations decreased the likelihood of social acceptance of people with schizophrenia and major depression. ${ }^{32}$

This study supports the evidence that knowledge of genetic susceptibility could carry potential for both health promotion and harm through genetic validation versus genetic discrimination, respectively. Further evaluation of public views with regard to the effect of predictive genetic testing for psychiatric disorders on the stigma is now required in a larger population. This is especially pertinent considering the current availability of DTC predictive genetic testing for allelic associations with various psychiatric disorders.

Voluntary reporting of a personal or family history of mental illness could be a limitation of the study as this may have resulted in the affected group only represented by those willing to disclose such information. Intention to have a genetic test shown in this study may not be a true indication of uptake of a predictive genetic test for a multifactorial disorder because uptake has been shown to be lower than intention to test. ${ }^{33}$

\section{CONCLUSIONS}

High interest in hypothetical predictive genetic testing for depression risk alleles, especially among individuals with a personal or family history of mental illness, suggests there would be a future demand for psychiatric genetic testing, potentially moderated by perceived discrimination and privacy issues. These findings highlight the need for legislation to minimise the risk of potential genetic discrimination resulting from predictive genetic testing in psychiatry. Given the relatively low risk rates for close family members for developing psychiatric disorders with incomplete penetrance compared with Mendelian inherited traits, risks should be kept in perspective when informing the public and designing mental health interventions. The role of environmental risk factors as well as heritability should be emphasized. These qualitative findings now require replication using a survey design in large representative non-clinical general population samples before recommendations about mental health interventions based on genetic risk can be made on a broader scale.

\section{ACKNOWLEDGEMENTS}

We thank Dr Nadine Kasparian for training in the use of QSR N6 software, Dr Catriona Bonfiglioli for advice on methodology and study participants who gave their time.This study was supported by Australian National Health and Medical Research Council (NHMRC) Public Health PhD scholarship (455414), NHMRC Career Development Award (350989) and NHMRC Program Grant (10135). 
1 Cannon TD, Cadenhead K, Cornblatt B et al: Prediction of psychosis in youth at high clinical risk: A multisite longitudinal study in North America. Arch Gen Psychiatry 2008; 65: 28-37.

2 Kraft P, Hunter DJ: Genetic risk prediction - are we there yet? N Eng/ J Med 2009; 360: 1701-1703.

3 Hudson K, Javitt G, Burke W, Byres P: ASHG statement on direct-to-consumer genetic testing in the United States. Am J Hum Genet 2007; 81: 635-637.

4 Prainsack B, Reardon J, Hindmarsh R, Gottweis H, Naue U, Lunshof JE: Personal genomes: misdirected precaution. Nature 2008; 456: 34-35.

5 Caspi A, Sugden K, Moffitt TE et al: Influence of life stress on depression: moderation by a polymorphism in the 5-HTT gene. Science 2003; 301: 386-389.

6 Taylor SE, Way BM, Welch WT, Hilmert CJ, Lehman BJ, Eisenberger NI: Early family environment, current adversity, the serotonin transporter promoter polymorphism, and depressive symptomatology. Biol Psychiatry 2006; 60: 671-676.

7 Wilhelm K, Mitchell PB, Niven $\mathrm{H}$ et al: Life events, first depression onset and the serotonin transporter gene. Br J Psychiatry 2006; 188: 210-215.

8 Kendler KS, Kuhn JW, Vittum J, Prescott CA, Riley B: The interaction of stressful life events and a serotonin transporter polymorphism in the prediction of episodes of major depression: a replication. Arch Gen Psychiatry 2005; 62: 529-535.

9 Eley TC, Sugden K, Corsico A et al: Gene-environment interaction analysis of serotonin system markers with adolescent depression. Mol Psychiatry 2004; 9: 908-915.

10 Kaufman J, Yang B-Z, Douglas-Palumberi H et al: Social supports and serotonin transporter gene moderate depression in maltreated children. Proc Natl Acad Sci USA 2004; 101: 17316-17321.

11 Risch $\mathrm{N}$, Richard $\mathrm{H}$, Lehner T et al: Interaction between the serotonin transporter gene (5-HTTLPR), stressful life events, and risk of depression: a meta-analysis. JAMA 2009; 301: 2462-2471.

12 Meiser B, Mitchell PB, McGirr H, Van Herten M, Schofield PR: Implications of genetic risk information in families with a high density of bipolar disorder: an exploratory study. Soc Sci Med 2005; 60: 109-118.

13 Phelan J: Genetic bases of mental illness - a cure for stigma? Trends Neurosci 2002 ; 25: 430-431.

14 Link BG, Yang LH, Phelan JC, Collins PY: Measuring mental illness stigma. Schizophr Bull 2004; 30: 511 .

15 Meiser B, Mitchell P, Kasparian $\mathrm{N}$ et al: Attitudes towards childbearing, causal attributions for bipolar disorder and psychological distress: a study of families with multiple cases of bipolar disorder. Psychol Med 2007; 37: 1601-1611.

16 Morley KI, Hall WD, Carter L: Genetic screening for susceptibility to depression: can we and should we? Aust NZ J Psychiatry 2004; 38: 73-80.

17 Human Genetics Commission UK: More Genes Direct: A Report on Developments in the Availability, Marketing and Regulation of Genetic Tests Supplied Directly to the Public. UK: Department of Health, 2007.
18 Australian Law Reform Commission, National Health and Medical Research Council (Australia), Australian Health Ethics Committee: Essentially Yours: The protection of human genetic information in Australia. Australian Law Reform Commission, 2003, pp 1158

19 Trippitelli CL, Jamison KR, Folstein MF, Bartko JJ, DePaulo JR: Pilot study on patients' and spouses' attitudes toward potential genetic testing for bipolar disorder. Am J Psychiatry 1998; 155: 899-904.

20 Smith LB, Sapers B, Reus VI, Freimer NB: Attitudes towards bipolar disorder and predictive genetic testing among patients and providers. J Med Genet 1996; 33: 544-549.

21 Jones I, Scourfield J, McCandless F, Craddock N: Attitudes towards future testing for bipolar disorder susceptibility genes: a preliminary investigation. J Affect Disord 2002; 71: 189-193.

22 DeLisi LE, Bertisch H: A preliminary comparison of the hopes of researchers, clinicians, and families for the future ethical use of genetic findings on schizophrenia. Am J Med Genet B Neuropsychiatr Genet 2006; 141: 110-115.

23 Meiser B, Kasparian NA, Mitchell PB et al: Attitudes to genetic testing in families with multiple cases of bipolar disorder. Genet Test 2008; 12: 233-244.

24 Wilde A, Meiser B, Mitchell PB, Schofield PR: Community attitudes towards mental health interventions for healthy people on the basis of genetic susceptibility. Aust NZ J Psychiatry, In press.

25 Kitto SC, Chesters J, Grbich C: Quality in qualitative research. Criteria for authors and assessors in the submission and assessment of qualitative research articles for the Medical Journal of Australia. Med J Aust 2008; 188: 243-246.

26 Stewart D, Shamdasani P: Focus Groups: Theory and Practice. Newbury Park, CA: Sage Publications Inc, 1990.

27 Patton M: Qualitative Evaluation and Research Method, 3rd edn, London: Sage Publications Inc, 2002.

28 Miles MB, Huberman AM: Qualitative Data Analysis: An Expanded Sourcebook, 2nd edn, London: Sage Publications, Inc, 1994.

29 Austin JC, Smith G, Honer WG: The genomic era and perceptions of psychotic disorders: genetic risk estimation, associations with reproductive decisions and views about predictive testing. Am J Med Genet B Neuropsychiatr Genet 2006; 141B: 926-928.

30 Phelan J: Geneticization of deviant behaviour and consequences for stigma: The case of mental illness. J Health Soc Behav 2005; 46: 307-322.

31 Austin JC, Honer WG: The potential impact of genetic counseling for mental illness. Clin Genet 2005; 67: 134-142.

32 Dietrich S, Matschinger $\mathrm{H}$, Angermeyer MC: The relationship between biogenetic causal explanations and social distance toward people with mental disorders: results from a population survey in Germany. Int J Soc Psychiatry 2006; 52: 166-174.

33 Lerman C, Croyle R, Tercyak K, Hamann H: Genetic testing: Psychological aspects and implications. J Consult Clin Psychol 2002; 70: 784-797. 